\title{
Système des acteurs et stratégie de formulation
}

Olivier LAPIERRE

INA P-G, Département des sciences animales Centre d'Etude et de Recherche sur I'Economie et l'Organisation des Productions Animales (CEREOPA), 16, rue Claude-Bernard,

F75231 Paris cedex 05, France

\begin{abstract}
Formulation is a central strategy for appropriate protein supply and use. It aims at combining the best answer to animal nutritional requirements together with the economic efficiency of the feed industry. To this end, it is necessary to consider the diverse sources of feed ingredients and to favor the substitution of one raw material by another one whenever it improves the technical or economical performances of the feed. This can be achieved by optimization, which is the key tool of animal feed formulation. The formulation strategy has the potential to respond to many other issues than the technical and economical ones, such as the environmental and safety issues. This wider approach allows us to better appraise the complexity of the production system, which encompasses many interacting agents, and to more efficiently evaluate their respective role and impact on the feed supply.
\end{abstract}

Key words: animal feeding, formulation, protein supply
La stratégie de formulation est, par nature, au cœur de la question des apports protéiques. Par-delà l'apparence de simplicité de l'exercice de mise au point d'une recette, la formulation se révèle être à la jonction d'un jeu de forces auxquels participent de nombreux acteurs. La figure 1 illustre cette interaction. Ce système d'acteurs intervient en amont du processus pour déterminer la situation des ressources alimentaires sur laquelle se base l'opération de formulation [1]. II intervient aussi en aval, quand, au-delà de la recette d'alimentation, qui est le résultat factuel de la formulation, c'est la performance visée qui est considérée.

Cet article se propose, après avoir rappelé ce que sont les principes de base de la formulation, de montrer en quoi le passage vers l'enjeu de la performance implique un système $d^{\prime}$ acteurs complexe et en quoi il est susceptible d'interférer sur la nature des approvisionnements des filières animales.

\section{La formulation : la recherche d'une solution d'alimentation}

La préoccupation de formulation est probablement aussi ancienne que la pratique de l'élevage. Activité raisonnée, elle s'impose dès lors que l'éleveur ne confie plus au seul instinct de ses animaux laissés en liberté le soin d'assurer leur alimentation. Les agronomes latins rangeaient déjà l'alimentation au nombre des principales compétences d'un bon éleveur [2]. Associé au problème d'alimentation, l'objectif de la formulation est de mettre à disposition des animaux, de façon quotidienne, l'ensemble des ingrédients qui vont leur permettre de satisfaire aux besoins de leur entretien et de leurs fonctions de production (lait, viande, œuf, etc.). Mais la pratique de formulation ne se résume pas uniquement au calcul d'une recette alimentaire. L'importance de l'enjeu

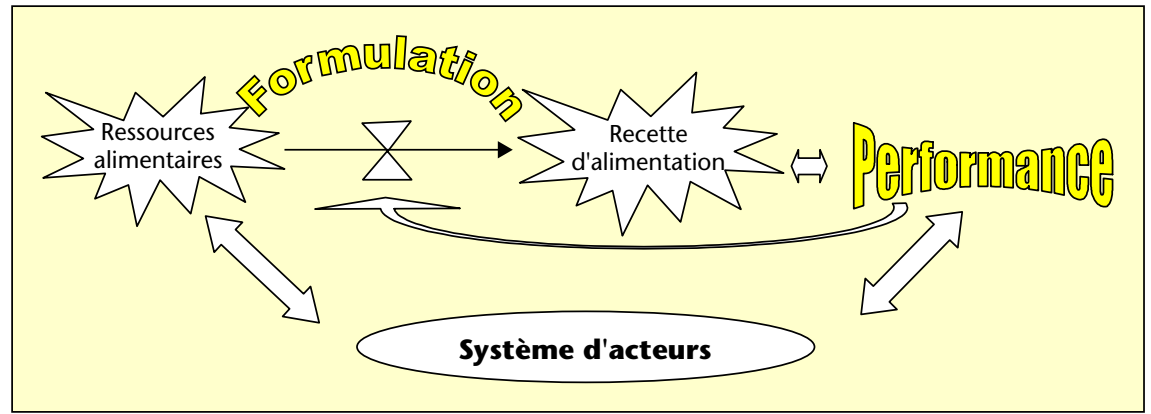

Figure 1. La formulation : une pratique ouverte sur un système d'acteurs. économique des choix d'alimentation fait que l'exercice de formulation va bien au-delà de la dimension technique. C'est la tentative de concilier ces deux exigences qui, très souvent, transforme la pratique de formulation en démarche d'optimisation.

\section{Le calcul d'une recette}

La mise au point d'une formule, d'une recette, est l'objectif premier de la formulation.

\section{Sur l'élevage ou chez le fabricant d'aliment} La première situation où s'exerce la pratique de la formulation est l'exploitation agricole sur laquelle sont détenus les animaux. Que ce soit par les règles de conduite du pâturage pour des herbivores où par le calcul de mélanges fermiers de matières premières pour un cheptel porcin, l'éleveur est, naturellement, le premier à être concerné par les choix d'alimentation de son cheptel. Ces décisions, il les prend seul ou avec l'aide de conseillers qui lui apportent les informations et procédures nécessaires. Le plus souvent il ne les remettra en cause qu'aux périodes où surviennent des changements majeurs dans le fonctionnement de son système de production : une ou deux fois par an. Le fabricant d'aliments composés, fournisseur des élevages, représente la seconde situation dans laquelle s'exerce la pratique de la formulation. Confronté comme l'éleveur à la question du choix des matières premières à incorporer dans le mélange qu'il mettra en marché, il accorde à cette décision une importance capitale. Dans un contexte où le nombre des matières premières potentiellement utilisable est élevé, son choix conditionnera grandement 
sa compétitivité et, par voie de conséquence, la performance de son entreprise. En prise avec un marché des matières premières volatil, il reviendra beaucoup plus fréquemment que l'éleveur sur sa décision. Actuellement, c'est au moins sur une base mensuelle que sont revues les formules calculées par les industriels de I'alimentation animale. Historiquement, ce secteur industriel a même été l'un des premiers à utiliser, en routine, les ordinateurs pour la mise en œuvre de cette opération [14].

\section{Individuelle ou collective}

Devant s'adapter à la situation de chaque animal, la formulation n'est cependant pas toujours réalisée à titre individuel. Selon la taille des cheptels exploités et selon les méthodes d'élevage pratiquées, la recette sera adaptée au cas particulier de chaque animal ou, au contraire, établie de façon uniforme pour un groupe d'animaux dont on considère les besoins comme équivalents. Dans ce dernier cas, l'éleveur table sur une capacité d'autorégulation partielle des animaux : ceux dont les besoins sont les plus faibles laissant à ceux dont les besoins sont supérieurs les quantités d'aliment dont ils $n^{\prime}$ ont pas besoin.

\section{Au-delà des ingrédients}

Même si le résultat de la formulation s'exprime sous la forme d'une recette où sont spécifiés les pourcentages ou quantités des différentes matières premières qui y sont incorporées, le raisonnement mis œuvre se soucie peu de la nature de ces matières premières. La qualité de la recette est moins dans la nature des ingrédients qui la composent que dans le niveau des caractéristiques nutritionnelles et alimentaires que ce mélange permet d'atteindre. Ce principe, qui revient à n'apprécier les matières premières qu'au travers de leur caractérisation par un système d'unités qui en exprime la valeur alimentaire, peut être qualifié de principe du « nutriment anonyme ». Il est celui qui prévaut aujourd'hui en matière de formulation des aliments du bétail [3]. Cette situation distingue radicalement la pratique de l'alimentation animale de celle que l'on rencontre en matière de formulation d'aliments pour l'homme où, au contraire, tout l'accent est mis sur la nature des ingrédients utilisés. Un yaourt aux fraises doit majoritairement contenir du lait et des fraises. En effet, en alimentation animale, les cas où l'intérêt est accordé à une matière première, pour ce qu'elle est, plutôt que pour ce qu'elle apporte sur le plan nutritionnel, sont devenus assez rares. Les vertus de l'avoine, en tant que telle, pour l'alimentation des reproducteurs et de l'orge, en tant que telle, pour I'alimentation des porcelets restent des exceptions qui confirment la règle. Les UGF (Unknown Growth Factors) des farines de poisson, qui ont conduit des industriels de l'alimentation animale à favoriser leur utilisation, sont une illustration de cette volonté d'explicitation de la qualité des matières premières. Initialement supposés manifester la présence de promoteurs de croissance, ils ont plutôt, depuis, été considérés comme la traduction de l'excellent équilibre nutritionnel du produit, équilibre qu'on pouvait par ailleurs atteindre en travaillant avec précision avec d'autres matières premières [5]. En privilégiant une représentation « fonctionnelle » des matières premières, le formulateur se met en position de laisser le jeu libre à toutes les possibilités de substitution. Pour lui, l'énergie nette du maïs équivaut à celle du soja, la lysine digestible $d u$ blé équivaut à celle du pois. L'intérêt des matières premières ne dépend plus que du rapport entre leur concentration nutritionnelle et les conditions économiques de leur disponibilité.

Cette explicitation de la qualité des matières premières, qui est garante des possibilités de substitution, s'est faite dans deux directions. Le nombre des critères utilisés s'est accru (on est passé, par exemple, de la teneur en protéine à la teneur en acides aminés qui constituent cette protéine) leur mode d'expression s'est sophistiqué de façon à représenter au plus près les processus de valorisation par les animaux (on est passé, par exemple, de la teneur en phosphore à la teneur en phosphore disponible). Cette amélioration des systèmes alimentaires permet d'approcher de façon de plus en plus fine et précise la question de l'alimentation des animaux d'élevage. Elle permet de composer des recettes dont les caractéristiques, correspondant le plus exactement aux besoins de I'animal cible, lui permettent d'exprimer tout son potentiel et évitent les gaspillages, sources $d$ 'inefficacité économique ou environnementale (en ajustant les apports au plus près des besoins on limite les rejets).

Le jeu des acteurs qui participent de la mise au point des connaissances nécessaires à cette pratique est déterminant de la place que sera susceptible d'occuper telle ou telle matière première. L'exemple récent de la révision des valeurs nutritionnelles et alimentaires des matières premières qui a accompagné la publication des nouvelles tables INRA/AFZ [4] en fournit une preuve indiscutable. L'application du seul changement des valeurs nutritionnelles induit par cette mise à jour s'est montrée très susceptible de modifier l'intérêt relatif des matières premières. En simulant les conséquences de ces changements, par le biais du modèle «Prospective Aliment " [6], on a pu montrer leur impact potentiel sur les utilisations des matières premières. II ressort de cette comparaison (figure 2) une baisse sensible des utilisations de tourteaux de tournesol métropolitain (- $393000 t$, soit - $68 \%$ ) et de colza (globalement, -202000 t, soit $-26 \%$ ) au profit du tourteau de soja (+ 537000 t, soit + $15 \%$ ). On assisterait également à un rééquilibrage dans les utilisations de sous-produits des céréales avec une hausse des incorporations de son (+ 195000 t, soit + $21 \%)$ aux dépens du remoulage (-248 $000 \mathrm{t}$, soit- $18 \%$ ). Enfin, les céréales comme le blé ou le sorgho verraient leurs utilisations progresser d'environ $100000 \mathrm{t}$ alors que celles du maïs reculeraient de $220000 \mathrm{t}$.

\section{Un enjeu économique}

La mise au point d'une formule ne représente cependant pas uniquement un apport nutritionnel. Sa signification économique est au moins aussi importante que sa signification technique.

\section{Le premier poste du coût de production}

L'importance du poste aliment dans le calcul du prix de revient des produits animaux confère à la formulation un rôle majeur dans le maintien de la compétitivité des filières animales. La production du poulet de chair constitue un bon exemple de cette affirmation. Les données de la figure 3 en fournissent une illustration très parlante. Même si la tendance va dans le sens d'une diminution du prix de l'aliment, celui-ci continue de représenter largement plus de $50 \%$ du coût de production total du poulet. C'est d'ailleurs le facteur aliment qui continue d'expliquer l'essentiel du différentiel de compétitivité qui existe entre la France et ses principaux concurrents en matière d'aviculture au stade de l'élevage.

\section{Un champ très concurrentiel}

Cet enjeu économique de la formulation est encore exacerbé par le fait que, sur le marché, la concurrence entre les entreprises qui produisent des aliments composés pour les éleveurs se fait le plus souvent sur la base du prix de vente. Dans ces conditions, des écarts de prix de revient, même minimes, sont susceptibles de faire basculer la décision de l'acheteur dans un sens ou dans l'autre. Cette sensibilité explique en partie le comportement des fabricants d'aliment qui attachent une importance majeure à leur politique d'achat, cherchant, par là, à limiter autant que possible le risque de se voir disqualifiés par un concurrent mieux placé qu'eux sur le marché des matières premières. L'importance de l'enjeu économique de la formulation se propage même en amont vers les fournisseurs de matières premières. Pour ces derniers, il est essentiel de savoir fixer une politique de prix qui leur permette de rester attractifs aux yeux de leurs clients. Nombre de ces acteurs d'amont, même s'ils n'ont pas à proprement parler de préoccupations en matière de production de mélanges alimentaires, se sont dotés de compétences en 


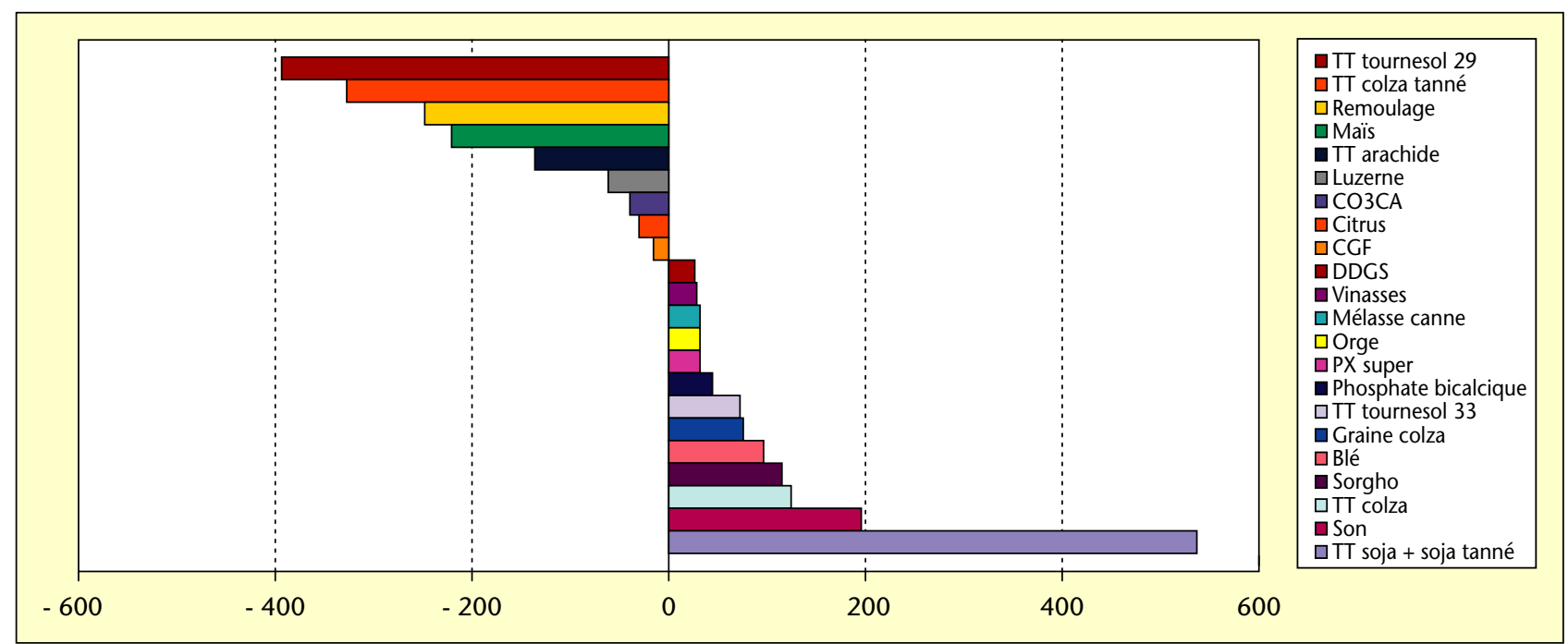

Figure 2. Comparaison des consommations potentielles de matières premières pour la campagne 2002-2003 entre les valeurs chimiques et nutritionnelles du modèle Prospective Aliment et les valeurs proposées par les Tables INRA/AFZ 2002 [5].

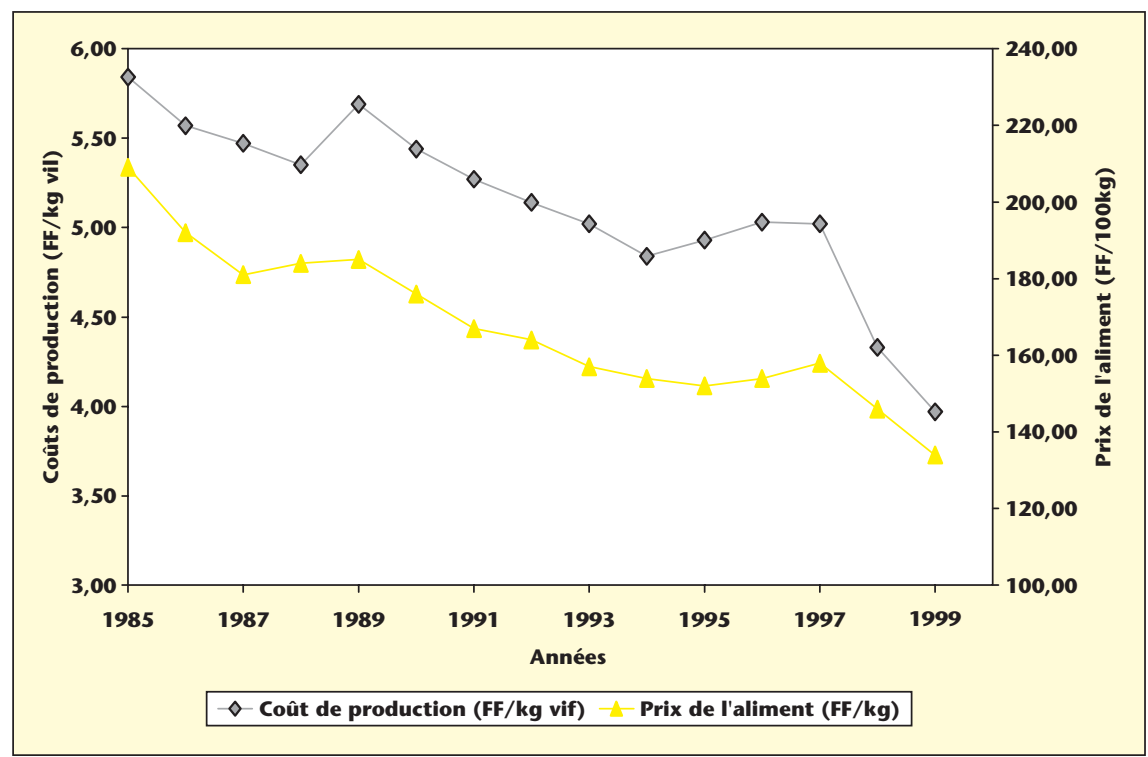

Figure 3. Importance du prix de l'aliment sur le coût de production du poulet de chair. Source Cereopa d'après ITAVI.

matière de formulation de façon à mieux comprendre les logiques de décision de leurs clients et à adapter leur politique commerciale en matière de prix et de qualité de leurs produits [15].

\section{Une démarche d'optimisation}

À la recherche d'une combinaison de matières premières qui satisfassent aux exigences des animaux et placés dans un contexte où la pression économique est forte, les formulateurs sont naturellement enclins à aborder la question sous l'angle de l'optimisation. Les formulateurs d'aliments composés ont du reste su très rapidement s'approprier la technique de recherche opérationnelle qu'est la programmation linéaire pour en faire l'instrument privilégié de leurs calculs de formules d'aliment. Dès le début des années 60 , les techniques mathématiques de l'optimisation, nées des travaux de Dantzig en 1947 [7], étaient déjà régulièrement utilisées par les fabricants $d^{\prime}$ aliments du bétail. Étrangement, cette approche par l'optimisation n'est pas encore totalement généralisée. En particulier, elle n'est que peu développée au niveau des élevages d'herbivores dont les rations ne sont encore très souvent calculées que sur les seules bases techniques.
Nombre d'éleveurs laitiers continuent d'appliquer des démarches de rationnement qui $\mathrm{n}^{\prime}$ intègrent pas la dimension économique. On peut penser que la pression économique, qui s'exerce actuellement sur ce secteur, modifiera un peu cette attitude.

\section{La performance : objectif de la formulation}

L'obtention d'une performance est le moteur de la formulation. Au-delà de la mise au point d'une solution d'alimentation, c'est bien un objectif plus global que vise le formulateur. La nature de cette performance visée est d'ailleurs à l'origine de l'interaction d'un système d'acteurs qui dépasse souvent le champ de l'élevage ou de l'entreprise d'aliment du bétail. On peut expliquer cet élargissement du champ comme étant la résultante d'un double mouvement. Ce double mouvement concerne à la fois les dimensions de la performance et l'échelle à laquelle elle s'apprécie.

Sur l'axe des dimensions, la nature des préoccupations intégrées par le formulateur a évolué $d^{\prime}$ une situation où la maîtrise technique et son impact économique étaient au cœur des préoccupations vers une situation ou leur importance se voit relativisée par la prise en compte d'autres aspects. La signification commerciale, environnementale ou sociétale de ses choix peut jouer un rôle dans sa décision.

Sur l'axe des échelles, on constate un élargissement du champ des préoccupations. Au-delà des frontières de l'unité de production, élevage ou fabrique d'aliment, le formulateur est susceptible de considérer les interactions qui peuvent exister entre sa décision et des niveaux 


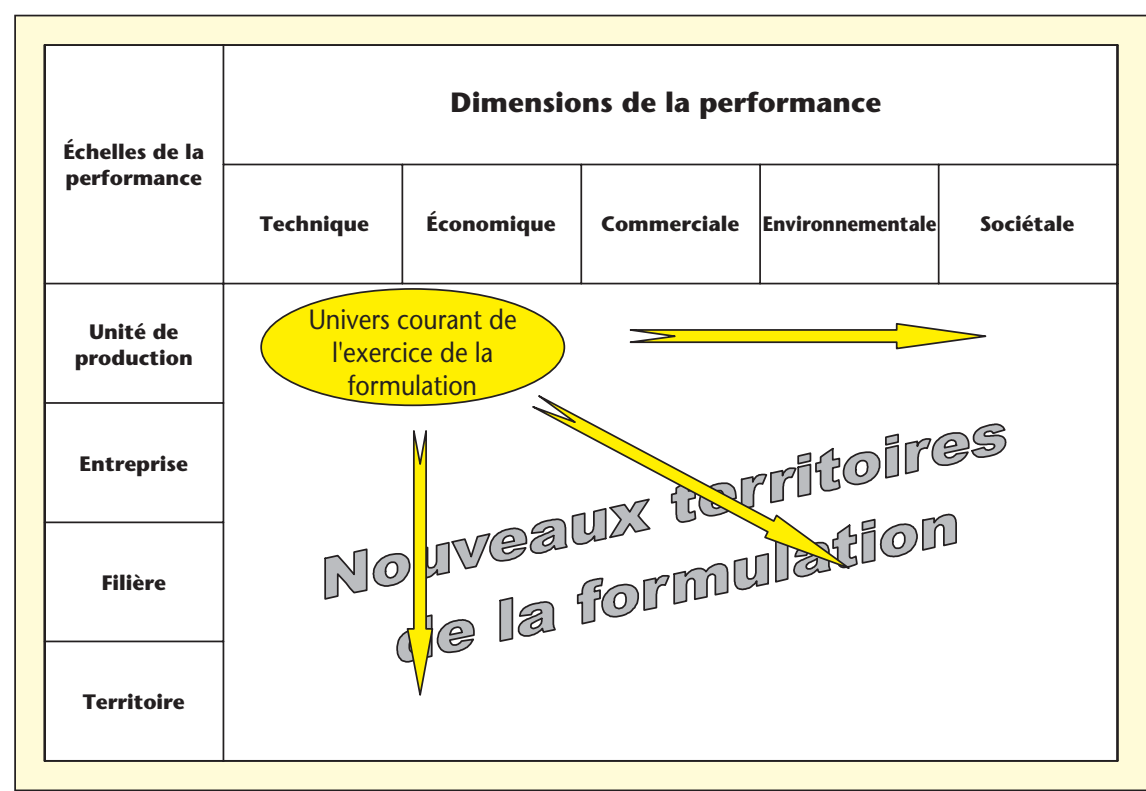

Figure 4. Les nouveaux territoires de la formulation. d'organisation supérieurs. L'entreprise, la filière voire le territoire deviennent ses nouveaux terrains de référence.

La figure 4 illustre ce changement de perspective auquel on assiste actuellement et qui devrait encore se développer à l'avenir.

\section{Différentes dimensions de la performance}

La multiplication des dimensions traitées par le formulateur est à l'origine d'une réelle complexification. Elle aboutit à la prise en compte dans le raisonnement de formulation de questions qui dépassent largement le seul champ de la satisfaction des besoins alimentaires des animaux.

\section{Dimensions techniques et économiques de la performance}

Les dimensions techniques et économiques restent celles auxquelles s'attache d'abord le formulateur pour optimiser le fonctionnement des systèmes de production. II s'agit en premier

lieu des animaux dont il va souvent chercher à maximiser les performances zootechniques. De nombreuses avancées de la zootechnie concernent cette compréhension de la relation animal/aliment. Elles ne seront pas traitées dans cet article. Mais il peut aussi s'agir des processus technologiques de stockage/ fabrication/distribution de l'aliment dont il va vouloir optimiser le rendement. Qu'ils soient ceux d'une unité industrielle de fabrication d'aliments composés ou ceux qui équipent une exploitation agricole, ils peuvent imposer des contraintes que le formulateur devra intégrer. Les contraintes de stockage sont, très souvent, des facteurs limitants majeurs [8]. Le tableau 1 illustre cet aspect dans un contexte de formulation d'aliment porc à la ferme.

\section{Dimension commerciale de la performance}

La dimension commerciale de la performance est une de celles que prend en compte le formulateur quand ses choix sont guidés par d'autres considérations que celles de la qualité

Tableau 1. Évolution du coût (€/q) et de la composition (\%) d'un aliment porcin fabriqué à la ferme en fonction du nombre de matières premières disponibles [8].

\begin{tabular}{|lccc|}
\hline Matières premières utilisables & $\mathbf{4}$ & $\mathbf{3}$ & $\mathbf{2}$ \\
\hline Blé & $52 \%$ & $55 \%$ & $79 \%$ \\
Tourteau de soja & $3 \%$ & $7 \%$ & $17 \%$ \\
Pois & $35 \%$ & $35 \%$ & - \\
Tourteau de colza & $6 \%$ & - & - \\
Minéraux & $2 \%$ & $2 \%$ & $2,5 \%$ \\
Lysine & - & - & $0,22 \%$ \\
Méthionine & - & $0,05 \%$ & $0,01 \%$ \\
Coût de l'aliment & $13,8 € / q$ & $14,3 € / q$ & $17,2 € / q$ \\
\hline
\end{tabular}

des matières premières et de leur prix. On en rencontre fréquemment deux exemples.

Le premier correspond à la situation dans laquelle les habitudes (culturelles?) de ses clients le contraignent à incorporer certaines matières premières «symboliques » dans leur mélange (voir ci-dessus) ou au contraire à éviter d'en incorporer d'autres, à la «mauvaise réputation ». La non-utilisation du tourteau de colza, en dépit de ses avantages économiques, reste un exemple de ce type de régulation. Dans un même ordre d'idée, on évoquera le choix qu'avait fait une importante firme de la grande distribution d'interdire l'utilisation des graines oléagineuses pour les produits animaux vendus sous sa marque.

Le second correspond à la situation des fabricants d'aliment qui ont mis en place une politique de collecte et valorisation des céréales produites sur les exploitations de leurs clients éleveurs. Cette politique s'était développée avant la réforme de la PAC de 1992 au titre des économies de taxes qu'elle permettait à l'éleveur de réaliser. Elle s'est poursuivie au-delà, même si l'avantage économique était devenu moindre, dans un contexte où les éleveurs restaient sensibles à la question de leur autonomie alimentaire et s'est même renforcée, suite à la crise de la vache folle, en tant que réponse au souhait de traçabilité. Pour le fabricant d'aliment, elle génère de la performance commerciale en ce qu'elle lui permet d'agrandir ou au moins de maintenir ses parts de marché en fidélisant son client. Elle lui impose, en contrepartie, d'accepter des niveaux d'incorporation de ces céréales que la seule performance technique et économique de l'élevage ne justifie pas toujours.

Même si elle n'apparait pas comme étant toujours très rationnelle, cette dimension commerciale a pris une importance croissante au cours des dernières années. Le fait que les entreprises y voient un moyen de segmentation de leur marché encourage probablement la tendance.

\section{Dimension environnementale de la performance}

La préoccupation environnementale a fait émerger cette question comme une dimension majeure du problème de formulation. Prise en compte en France plus tardivement que dans d'autres pays, la problématique de maîtrise des rejets a des répercussions directes sur les pratiques de formulation. Elle conduit à utiliser des systèmes d'appréciation de la qualité des matières premières qui traduisent mieux leur assimilation par les animaux. Elle conduit aussi au développement de technologies (traitements physico-chimiques ou biologiques) qui améliorent I'utilisation digestive des nutriments. Elle a poussé les formulateurs à resserrer le jeu des contraintes de façon à limiter les 


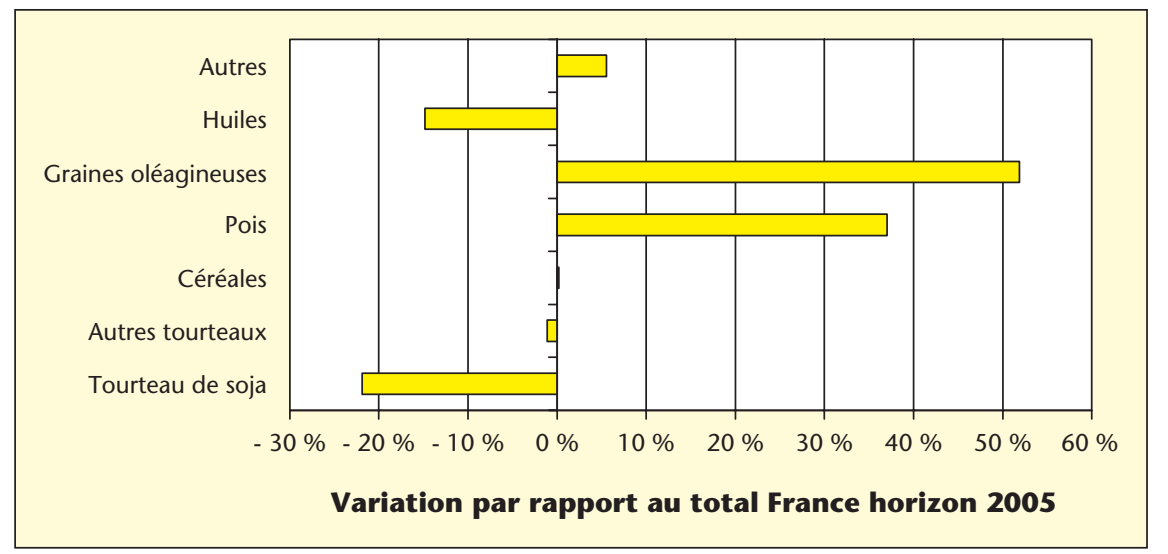

Figure 5. Conséquence de l'application de mesures agri-environnementales sur I'utilisation des matières premières dans les aliments composés pour volailles [9].

risques de gaspillage nutritionnel synonyme de pollution.

Les effets de la prise en compte de cette dimension dans la pratique de formulation sont sensibles sur le bilan d'approvisionnement en matières premières. Les résultats, obtenus dans le cadre d'une étude sur l'autonomie protéique des filières animales françaises [9], montrent (figure 5) que, sur le segment des aliments composés pour volailles, la prise en compte de ces contraintes modifie l'équilibre entre les matières premières protéiques et aboutit à réduire la consommation de tourteau de soja au profit du pois protéagineux et des graines oléagineuses.

On peut aussi rattacher à cette dimension les conséquences que pourrait avoir le fait que de nombreux agriculteurs envisagent aujourd'hui de développer des cultures énergétiques dans le but d'améliorer leur autonomie énergétique. Une telle option, si elle devait se confirmer, aurait des conséquences sur les ressources alimentaires disponibles sur les exploitations agricoles (tourteaux gras en particulier).

\section{Dimension sociétale de la performance}

L'évolution des attentes de la société à l'égard des activités de productions animales ne s'est pas seulement exprimée sur les questions environnementales. D'autres sujets ont émergé dont les conséquences sont aussi susceptibles $d$ 'influencer les pratiques de formulation.

La prise en compte du bien-être des animaux ne concerne pas simplement la taille de l'espace qui leur est alloué, la nature des sols sur lesquels ils circulent où l'environnement qui leur est proposé. Les spécialistes de ces questions travaillent actuellement sur les caractéristiques physiques des régimes et sur les effets induits en matière de comportement alimentaire. Ces travaux pourraient aboutir à la prise en compte de nouvelles contraintes nutritionnelles telles que, par exemple, la satisfaction fait adaptées pour aborder les questions de cohérence des choix entre production végétale et production animale. Le modèle d'optimisation PerfAgro, développé par le CEREOPA, permet de « calculer » des solutions optimales au sein d'un système où coexistent des activités de production végétale et des activités d'élevage. Les solutions, obtenues dans une optique de maximisation de la marge globale, proposent des orientations en matière $d^{\prime}$ 'assolement, d'une part, de stratégie d'alimentation des ateliers animaux, d'autre part. Son utilisation la plus naturelle est celle qui permet de déterminer la combinaison optimale des activités de production végétale dans une perspective de valorisation de leur produit pour l'approvisionnement en matières premières d'activités d'élevage. II permet alors de calculer un assolement et des rations ou formules optimaux. L'approche permet de prendre en compte les spécificités techniques des systèmes, elle intègre les contraintes relatives à la mise en œuvre de la nouvelle PAC et permet de gérer des contraintes telles que la main-d'œuvre disponible. Elle détermine une solution optimale en fonction des hypothèses économiques retenues par I'utilisateur. Actuellement appliqué aux cas des productions de bovins lait, ovins viande et porcins, un tel modèle peut prendre en compte de façon simultanée ou pas tous les types de productions animales présents dans la situation traitée. Les résultats du tableau 2 sont une illustration de cette utilisation au cas d'une exploitation agricole, la ferme expérimentale de I'INA P-G à Grignon, dans I'optique d'une évaluation des conséquences de la réforme de la PAC.

\section{Différentes échelles de la performance}

Les choix associés à la solution d'alimentation concernent d'abord le niveau de l'unité de production, l'élevage et le process de production d'aliment. Néanmoins, des éléments relevant d'autres échelles de fonctionnement sont susceptibles d'être pris en compte.

\section{L'échelle de l'entreprise}

Plus englobante que l'échelle de l'unité de production, celle de l'entreprise peut parfois être prise en considération pour apprécier la performance d'un choix de formulation. Ce sera en particulier le cas quand l'éleveur, au-delà de la question de l'alimentation de son cheptel, tiendra compte de l'intérêt de l'assolement de l'ensemble de son exploitation agricole. II pourra alors avoir à arbitrer ses choix non seulement en fonction de l'intérêt que telle production végétale présente en tant que ressource alimentaire mais aussi en fonction de ce que sa mise en culture empêche de développer comme autre production pour laquelle le marché peut s'avérer particulièrement intéressant. Les démarches d'optimisation habituellement utilisées pour traiter les questions de formulation des mélanges alimentaires sont alors tout à

\section{L'échelle de la filière}

En aval de l'élevage, la filière est un autre champ d'appréciation de la performance induite par les choix alimentaires. C'est aussi un niveau à partir duquel peuvent s'exprimer des contraintes justifiées par des enjeux technologiques et commerciaux.

Les exigences des technologies de transformation des produits animaux peuvent imposer à ces derniers d'être dotés de caractéristiques qui dépendent, en partie, des choix d'alimentation. C'est ainsi que les objectifs de quantité et de qualité du gras des carcasses de porcs valorisées dans la filière du « Jambon de Bayonne » se répercutent sur les contraintes de l'alimentation. Par la spécification de limites sur la teneur en acides gras des aliments utilisés, cette exigence de la filière limitera le choix des matières premières utilisables. L'industrie laitière, à la recherche de lait pour la production fromagère, sera elle aussi susceptible de faire remonter de l'aval des contraintes sur les objectifs de rationnement des troupeaux laitiers. Cela pourra concerner la teneur en protéine du lait et avoir pour conséquence de favoriser l'usage 
Tableau 2. Un modèle d'optimisation global à l'interface des choix de production végétale et de production animale (Application au cas de la ferme expérimentale de I'INA P-G à Grignon : exploration des conséquences de la réforme de la PAC sur la stratégie de formulation de l'étable laitière).

\begin{tabular}{|c|c|c|c|c|}
\hline \multicolumn{5}{|c|}{ 1. Assolements optimisés } \\
\hline Assolement (ha) & \multicolumn{2}{|c|}{ Aprés réforme } & \multicolumn{2}{|c|}{ Avant réforme } \\
\hline Blé oublé & \multicolumn{2}{|c|}{0} & \multicolumn{2}{|c|}{0} \\
\hline Blé assolé (dont semence) & \multicolumn{2}{|c|}{171,2} & \multicolumn{2}{|c|}{150,8} \\
\hline Colza & \multicolumn{2}{|c|}{15,7} & \multicolumn{2}{|c|}{43,8} \\
\hline Escourgeon (dont semence) & \multicolumn{2}{|c|}{68} & \multicolumn{2}{|c|}{80,3} \\
\hline Féverole $($ Smax $=20)$ & \multicolumn{2}{|c|}{20} & \multicolumn{2}{|c|}{20} \\
\hline Jachère (Smin = 50) & \multicolumn{2}{|c|}{50} & \multicolumn{2}{|c|}{50} \\
\hline Luzerne & \multicolumn{2}{|c|}{23,2} & \multicolumn{2}{|c|}{0} \\
\hline $\begin{array}{ll}\text { Maïs } & \text { Smax }=150 \\
\text { fourrage } & \end{array}$ & \multicolumn{2}{|c|}{46,8} & \multicolumn{2}{|c|}{61,1} \\
\hline Maïs grain & \multicolumn{2}{|c|}{103,2} & \multicolumn{2}{|c|}{88,9} \\
\hline Prairie $(S \min =33)$ & \multicolumn{2}{|c|}{33} & \multicolumn{2}{|c|}{33} \\
\hline \multicolumn{5}{|c|}{ 2. Rations optimisées } \\
\hline \multirow[t]{2}{*}{ Ratios des vaches (en Kg/jour) } & \multicolumn{2}{|c|}{ Après réforme } & \multicolumn{2}{|c|}{ Avant réforme } \\
\hline & VL1 & VL2 & VL1 & VL2 \\
\hline Colza graine & 0,69 & 0,13 & & 0,03 \\
\hline Craie & & 0,12 & & \\
\hline Drèches (MS) & 1,30 & 1,30 & 1,30 & 1,30 \\
\hline Foin $1^{\text {re }}$ coupe (MS) & 0,75 & 1,27 & 1,17 & 1,83 \\
\hline Foin $2^{\mathrm{e}}$ coupe (MS) & & 1,11 & 1,11 & \\
\hline Luzerne foin (MS) & 6,77 & & & \\
\hline Maïs fourrage (MS) & 10,00 & 10,34 & 15,36 & 11,04 \\
\hline Maïs grain & 4,27 & 1,86 & 0,98 & 1,42 \\
\hline Phosphate bicalcique & 0,01 & & 0,18 & 0,17 \\
\hline Pulpes surpressées (MS) & & 3,00 & 0,75 & 3,00 \\
\hline Tourteau de colza & 1,97 & 1,56 & 3,84 & 2,25 \\
\hline Tourteau de colza tanné & 0,21 & 1,56 & 1,16 & 1,45 \\
\hline Urée & & 0,02 & & \\
\hline
\end{tabular}

de matières premières susceptibles de l'améliorer. L'introduction de méthionine protégée dans les aliments peut résulter de cette préoccupation. Mais cela peut aussi concerner les qualités sanitaires du lait et justifier l'interdiction de matières premières jugées potentiellement vectrices de contamination. Ce sera, par exemple, l'exemple de l'interdiction de I'ensilage de maïs dans certaines filières de fromages $d^{\prime} A O C$.

Les exigences commerciales des filières sont aussi des motifs pour faire remonter, vers le formulateur, des contraintes que la seule performance de l'élevage ne nécessite pas. C'est ainsi que les filières de volailles sous signe de qualité, Label Rouge en particulier, ont imposé des seuils minimaux d'incorporation de céréales. Justifiée pour des raisons d'image, cette exigence est lourde de conséquences sur la nature des matières premières protéiques qui peuvent être utilisées pour rééquilibrer les mélanges alimentaires. Les résultats obtenus dans le cadre d'une étude conduite en 2001 [9] ont permis de montrer que l'élargissement de la contrainte d'un minimum de $75 \%$ de céréales pour un aliment volaille sous label à une contrainte de $75 \%$ minimum de céréales et pois protéagineux permettait d'économiser de tourteau de soja. Les positions prises par les filières à l'égard des OGM sont d'une nature comparable. En s'interdisant de mettre en marché des produits animaux provenant de systèmes dans lesquels des OGM auraient été incorporés dans les aliments, certains opérateurs de l'aval des filières ont profondément influencé la nature des ressources protéiques utilisées. Quand les acteurs n'ont pas pris le parti de n'utiliser que du soja sans OGM (soja tracé en provenance du Brésil ou soja non OGM du sud de la France) elles ont alors dû prendre celui $d^{\prime}$ 'éviter cette matière première et de se tourner vers d'autres matières premières riches en protéines.

Comme précédemment la dimension commerciale, on a le sentiment que cette échelle de la filière est en train de prendre une grande importance. Cela peut s'expliquer par le fait qu'un tel élargissement peut être le support d'une politique de différenciation vers laquelle se tournent de nombreuses entreprises qui y voient une façon d'échapper à une pression concurrentielle devenue très dure sur le champ des produits ordinaires. annuellement l'équivalent de 35000 tonnes

\section{L'échelle du territoire}

Dernière échelle à laquelle peut se traiter la question de la performance, celle du territoire n'est probablement pas encore la plus prise en compte. On perçoit quand même, ici ou là, les prémices de son émergence dans le champ de la formulation.

C'est déjà à cette échelle qu'en 1978, le gouvernement français décidait de la mise en place d'un «Plan protéines» dont l'enjeu était la reconquête de l'autonomie protéique du système d'élevage national [11]. II n'est pas nécessaire de rappeler les répercussions de cette décision politique sur l'évolution du bilan d'approvisionnement en matières premières protéiques des filières animales françaises. C'est aussi l'abandon de cet enjeu, à l'échelle d'un territoire encore plus vaste, celui de I'Europe, qui aboutit actuellement à une dégradation relative de la performance « autonomie protéique » de I'UE. C'est également à cette échelle du territoire qu'une préoccupation de performance sanitaire avait conduit à restreindre puis définitivement interdire l'incorporation des co-produits animaux dans les aliments du bétail. Là encore, les conséquences en terme d'apports protéiques ont été considérables.

Dans un autre domaine, c'est encore cette échelle qui est concernée par le choix de la filière " Jambon de Bayonne » de privilégier le recours à des matières premières produites localement pour alimenter ses porcs [12]. C'est aussi cette perspective d'efficacité régionale qui a poussé la filière du Poulet de Loué à promouvoir la stratégie « grains de terroir » qui vise à encourager l'utilisation des céréales produites dans la zone de production des volailles.

Il est raisonnable d'envisager que, demain, les stratégies de formulation intègreront mieux les synergies qu'elles pourront contribuer à développer au niveau du territoire entre productions animales et productions de ressources alimentaires. Une politique territoriale de développement de la production de biocarburants ne saura se montrer performante que dans la complémentarité qu'elle trouvera auprès des filières animales en matière de valorisation de ses co-produits. Les résultats de simulations effectuées à partir du modèle « Prospective Aliment » du Céréopa (figure 6) ont montré l'importance d'une évolution des stratégies de formulation dans la perspective de l'augmentation de la production de biocarburants [13]. L'économie du modèle « Diester » est sensible au prix de valorisation du tourteau de colza. Or ses perspectives de développement devraient aboutir à la mise en marché de quantités supplémentaires de tourteaux très importantes. L'efficacité économique de l'opération ne sera garantie que si les stratégies de formulation peuvent garantir des perspectives suffisantes à I'utilisation de cette matière première. 


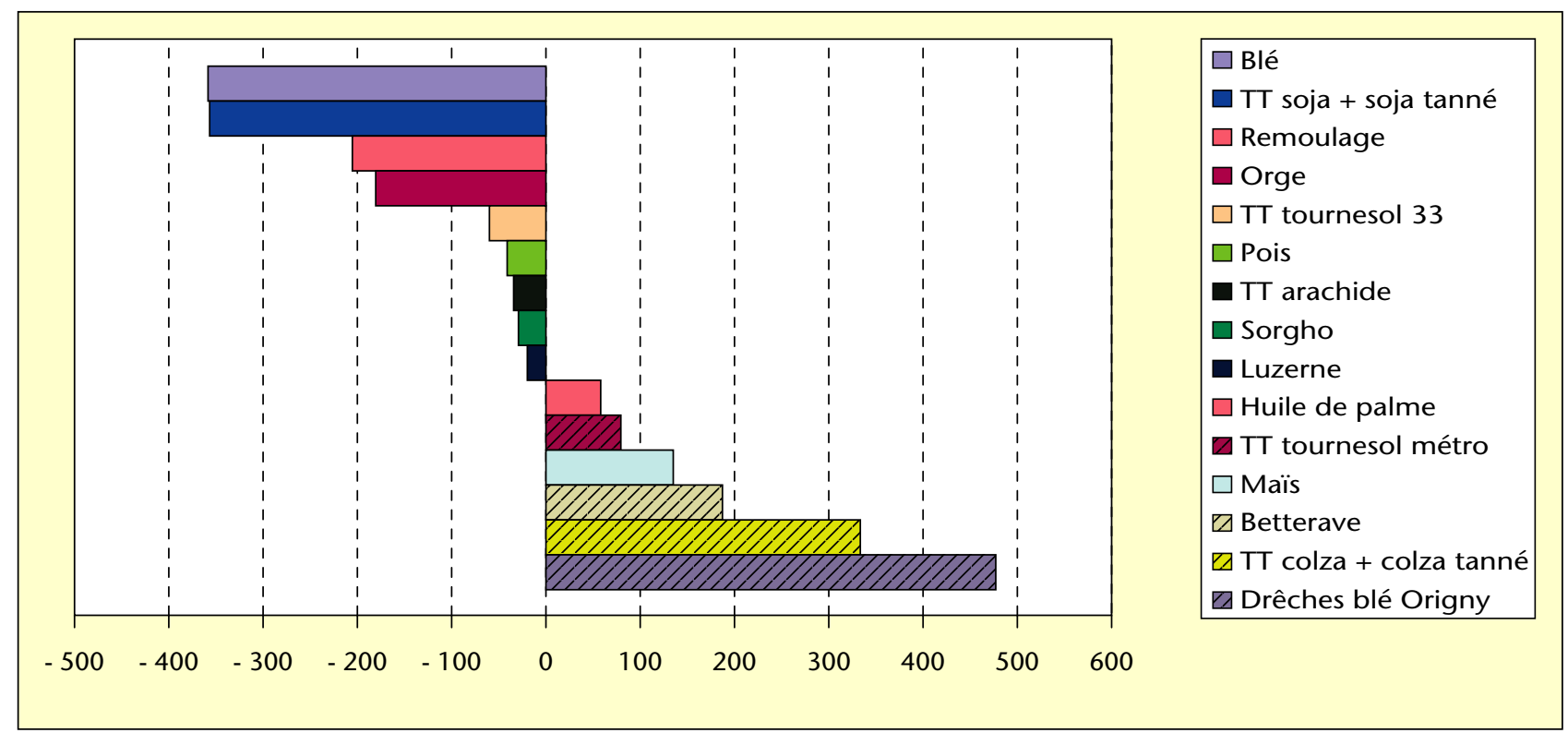

Figure 6. Conséquences de l'introduction de drêches de blé et des co-produits de la filière biocarburants sur les utilisations (en 1000 tonnes) des autres matières premières par les fabricants d'aliments composés - Scénario 2007 - Prix moyens [4].

\section{Conclusion}

La stratégie de formulation est d'abord le résultat de la mise en œuvre, au niveau d'une unité de production, d'un raisonnement technique et économique. C'est dans ce contexte que la démarche s'est sophistiquée pour faciliter une prise en compte plus précise du besoin des animaux et, par une meilleure caractérisation des matières premières, faciliter les substitutions sources majeures de l'efficacité économique recherchée par les filières animales. C'est aussi dans ce contexte qu'elle se fonde sur la mise en œuvre de procédures sophistiquées d'optimisation qui facilitent le calcul de recettes d'alimentation performantes.

Mais la performance des choix d'alimentation ne doit pas s'apprécier que localement. Leur efficacité ils la trouvent aussi sur d'autres registres que ceux de la technique et de l'économique et à d'autres échelles que celle de l'unité de production. Par cet élargissement de sa perspective, la formulation se trouve en interaction avec un système d'acteurs beaucoup plus complexe et se voit obligée de repenser la question de l'appréciation de son efficacité. La bonne formule alimentaire n'est plus uniquement celle qui fournit les meilleures performances d'élevage. Elle est aussi celle qui anticipe une attente des clients et fournit à la société les éléments de réponses qu'elle attend. Son efficacité ne se mesure plus au seul niveau de l'élevage mais aussi à celui de l'entreprise et de la filière auxquelles participe cet élevage ainsi qu'à celui du territoire où il est implanté. Ce changement de perspective pose alors la ques- tion de la coordination entre différentes catégories d'acteurs dont les références et modes de pensée ne sont pas toujours compatibles.

\section{RÉFÉRENCES}

1. LAPIERRE O. CULTURE ET ÉlEVAGE : quelles relations quelles synergies? OCL 2004; $11(4 / 5):$ 261-7.

2. RISSE. Histoire de l'élevage français. Paris : Editions de l'Harmattan, 1994 ; (365 pages).

3. LAPIERRE O. La formulation des aliments composés s'adapte aux nouvelles exigences. Oléoscope 79 : 10-1.

4. LAPIERRE O, PRESSENDA F. Évaluation de I'impact d'une augmentation de la disponibilité de drêches de distillerie de blé et autres coproduits de la filière biocarburants sur les utilisations de matières premières dans les aliments composés. Céréopa. Rapport d'étude, 2004 : (24 pages).

5. WINDSOR ML. FISH MEAL. Department of Trade and Industry, Torry Research Station. Torry Advisory Note No. 49, 2001 (Site Web de la FAO: http://www.fao.org/wairdocs/tan/ x5926e/ x5926e00.htm\#Contents).

6. CÉRÉOPA. Un éclairage original sur la consommation des matières premières en alimentation animale. CEREOPA : Présentation du modèle Prospective Aliment. 2000 (Site WEB du CEREOPA : http ://www.cereopa.com/pres modelefrance.htm).

7. DANTZIG GB. Applications et prolongements de la programmation linéaire. Paris: Dunod (traduit par E. Ventura), 1966 ; (433 pages).
8. PRESSENDA F, LAPIERRE O. Reconquête de I'autonomie protéique : quelles stratégies pour les productions porcines? Journées de la Recherche Porcine 2003 ; 35 : 83-8.

9. PRESSENDA F, LAPIERRE O. Reconquête de I'autonomie protéique : quelles stratégies pour les productions avicoles? Journées de la Recherche Avicole. 2003 ; 9-14.

10. SCHMIDELY $P$, LAPIERRE $O$, REMEUF $F$, MARTIN N, SAADE M, DUVAUX PONTER C. Soja vs colza sur la ferme de Grignon: quels risques pour les qualités nutritionnelles et organoleptiques des produits laitiers et quels avantages économiques? Deuxièmes rencontres de I'INAPG. 2003 ; (Paris (FRA), 2p).

11. DELPLANCKE D, LAPIERRE O. L'approvisionnement européen en protéines: un handicap relatif ? OCL $1998 ; 5(4)$ : 255-61.

12. KERVENO Y. Le Sud-Ouest en quête d'autonomie protéique. Réussir Porcs $2005 ; 117$ : $72-4$.

13. LAPIERRE O, PRESSENDA F. Analyse des répercussions de la publication des Tables INRA/AFZ 2002 sur les pratiques de formulation du secteur de l'alimentation animale. Céréopa. Rapport d'étude. 2004 ; (18 pages).

14. LAPIERRE O. Les nouvelles pratiques de formulation. Revue de I'Alimentation Animale 2000 ; $534: 45-8$.

15. LAPIERRE O. Stratégies de segmentation de I'offre en matières premières pour les marchés de I'alimentation animale. Valoriscop. Janvier 2001 ; (Paris. 17 et 18). 